\title{
Soziale Konsequenzen der Abschaffung des Vorruhestands für Langzeitarbeitslose
}

\author{
Tatjana Mika \\ Jochen Baumann
}

\begin{abstract}
Die Abschaffung der Frühverrentung und die Absenkung des Rentenniveaus haben die Alterssicherung aus der gesetzlichen Rente für langjährig Arbeitslose deutlich verändert. Der Beitrag veranschaulicht die Auswirkungen der Rentenreformen, indem er am Beispiel von identischen Musterbiografien die Einkommen aus gesetzlicher Rente des Geburtsjahrgangs 1939 mit den erwartbaren Renten des Jahrgangs 1955 vergleicht. Hierbei wird deutlich, wie sehr sich die sozialrechtliche Bewertung des Lebenssachverhalts dauerhafter Arbeitslosigkeit im Lebensjahrzehnt vor Eintritt in die Rente inzwischen gewandelt hat.
\end{abstract}

\section{Sozialpolitische Funktion der Frühverrentung}

Die sozialstaatliche Sicherung für ältere Arbeitslose wurde in den vergangenen Jahren radikal abgebaut. Damit bricht auch eine Brücke zusammen, die über einen Zeitraum von zwanzig Jahren hinweg älteren Arbeitslosen einen sozialpolitisch gesicherten Übergang aus der letzten Beschäftigung bis zur Rente ermöglicht hat. Die Sicherungspfeiler reichten vom Arbeitslosengeld über die Arbeitslosenhilfe bis in die vorgezogene Rente. Dieser institutionalisierte Vorruhestand bildete eine eigenständige biografische Phase für einen bedeutenden Anteil der älteren Beschäftigten und wurde dadurch zu einem charakteristischen Element des deutschen Modells der sozialstaatlichen Sicherung (Buchholz et al. 2006, S. 74f.). Bereits seit dem Jahr 2000 wird die Frühverrentung nun schrittweise abgebaut. Die Folge ist, dass viele Betroffene einer neuen, unsicheren Lebensphase zwischen deutlich verkürztem Arbeitslosengeldbezug und wesentlich später möglichem Eintritt in die Rente entgegensehen.

Der schrittweise durchgeführten, in der Gesamtwirkung aber radikalen Veränderung liegen die - aus der Perspektive der Betroffenen - gleichzeitig wirksam werdenden Reformen der Arbeitslosen- und Rentenversicherung zugrunde. Mit Blick auf die Rentenversicherung wurde bereits 1992 vom Gesetzgeber beschlossen, dass die steigende Lebenserwartung und die damit einhergehenden demografischen Verschiebungen eine Erhöhung des Verrentungsalters erfordern. Die Möglichkeit, wegen Arbeitslosigkeit vorzeitig in Rente zu gehen, wurde für ab 1952 Geborene abgeschafft. Das Vorhaben, das Renteneintrittsalter behutsam anzuheben, wurde durch die Rentenreform 1996 beschleunigt und 2001 modifiziert, weshalb für einige Betroffene die Folgen sehr kurzfristig eintraten (Brall et al. 2004, S. 359). Die sozialen Auswirkungen zeigen sich im Kohortenvergleich besonders deutlich. Ingesamt wurde durch die Fülle der Rentenreformen ein sich ständig veränderndes Recht mit einer Reihe von Ausnahmen geschaffen, das eine klare Lebensplanung bezüglich des Übergangs in den Ruhestand für die Alterskohorten zwischen 1939 und 1955 erschwert. Die biografische Lücke, die durch die steigende Altersgrenze der Rentenversicherung entstand, ist nicht geschlossen worden, was über die Arbeitslosenversicherung möglich gewesen wäre.

\section{Vorruhestand als Ausweg aus Arbeitslosigkeit}

Wenn dem Renteneintritt eine Phase der Arbeitslosigkeit vorausgeht, so wird der Übergang in die Rente meist positiv erlebt. Hierfür ist nicht allein die Absicherung des Einkommens ursächlich. Vielfach steigt die Lebenszufriedenheit generell nach dem Übergang in den Ruhestand. Noch stärker ist dieser Effekt natürlich bei denjenigen, die vor der Rente arbeitslos waren (TeschRömer et al. 2006, S.421f.). Für sie markiert der Bezug einer Rente den Übergang in die gesellschaftlich anerkannte Lebensphase des legitimen und ausreichend ausgestatteten Ruhestands.

Historisch gesehen war der mit 60 Jahren mögliche, vorgezogene Rentenbezug wegen Arbeitslosigkeit zunächst ein Privileg der Angestellten (Albrecht/Müller 1996, S.123). Mit der Angleichung der Arbeiterund Angestelltenrentenversicherung auf dem Niveau der Angestelltensicherung kam es 1957 zur Ausweitung dieser Rentenart auch auf die Arbeiter (Schmähl 2007, S. 20). Infolge der Massenarbeitslosigkeit seit den späten 1980er Jahren und nochmals dynamisiert durch die ansteigende Arbeitslosigkeit in Ostdeutschland nach der Wiedervereinigung wurde im Jahr 1992 vom Gesetzgeber diese Rentenart so häufig in Anspruch genommen, dass das durchschnittliche Rentenalter messbar sank (Fasshauer 2005, S. 75). Um diesen Trend umzukehren, wurde die Abschaffung dieser Rentenart beschlossen, allerdings ursprünglich mit langfristiger Übergangszeit bis 2014 (Ruland 2007, S. 33f.).

Der sozialstaatlich abgesicherte Übergang aus der Arbeitslosigkeit in die Rente, der für die Rentenversicherung zu einem wachsenden Kostenfaktor wurde, hatte für die Gruppen, deren Chancen auf Wiedereinstellung bei Arbeitslosigkeit sehr gering waren, in den letzten zwei Jahrzehnten herausragende biografische Bedeutung. Dies

\footnotetext{
Tatjana Mika, Wissenschaftliche Referentin am Forschungsdatenzentrum der Rentenversicherung. Arbeitsschwerpunkte: Alterssicherung, empirische Gerechtigkeitsforschung. e-mail: Tatjana.Mika@drv-bund.de Jochen Baumann, Doktorand und Lehrbeauftragter am Institut für Sozia/wissenschaften der Humboldt-Universität zu Berlin. Arbeitsschwerpunkte: Europäische soziale Sicherung, Migration und soziale Ung/eichheit, Staatsbürgerschaft. e-mail: jochen.baumann@rz.hu-berlin.de
} 
traf und trifft für diejenigen besonders zu, die im Zuge der Umstrukturierung von Betrieben oder Branchen entlassen wurden und mit ihrer Qualifikation und Arbeitserfahrung auf dem (regionalen) Arbeitsmarkt kein neues Arbeitsangebot erhielten (Buchholz et al. 2006, S. 69). Eine solche Umstrukturierung traf besonders Ostdeutsche nach 1990, sodass es nach 1992 zunehmend kaum noch über 55-jährige Beschäftigte in ostdeutschen Betrieben gab (Wübbeke 2005a, S. 4). Speziell ostdeutsche Frauen der Alterskohorte um 1939 wurden nach der Wiedervereinigung besonders häufig und lange arbeitslos, weil bei ihnen eine durchschnittlich niedrigere Qualifikation mit einem für die Wiedereinstellung nach Arbeitslosigkeit bereits ungünstig hohen Alter zusammentraf (Trappe 2006, S. 133). In Westdeutschland gab es viele Betroffene unter den un- und angelernten Industriearbeitern, darunter viele Zuwanderer aus den ehemaligen Anwerbestaaten (Statistisches Bundesamt 2006, S. 102f.). So stieg die Arbeitslosenrate unter ausländischen Arbeitnehmern in den gesamten 1980er und 1990er Jahren bei insgesamt anwachsender Arbeitslosigkeit immer besonders schnell und besonders stark an und lag in etwa doppelt so hoch wie die deutsche Rate (Seifert 2000).

Etwa die Hälfte ihres Erwerbslebens verbrachte die 1939er Alterskohorte in den 1960er und 1970er Jahren und hatte in dieser Zeit gute Chancen auf durchgehende Beschäftigung auf dem westdeutschen Arbeitsmarkt. Erst in der zweiten Hälfte ihrer Biografie, in den 1980er und 1990er Jahren, kam es zu ansteigender Arbeitslosigkeit. Arbeitsmigranten waren von diesen Prozessen wegen ihrer Alters- und Beschäftigungsstruktur insgesamt stärker betroffen als Deutsche. Auf die erste Periode der Massenarbeitslosigkeit reagierte die damalige Bundesregierung mit speziellen Rückkehrförderungsprogrammen für ausländische Arbeitnehmer, die dazu motivieren sollten, den Arbeitsplatz aufzugeben. Häufig wurde daraufhin auch die betriebliche Personalpolitik, im Einvernehmen mit dem Betriebsrat, besonders darauf ausgerichtet, ausländischen Arbeitnehmern den Abschluss einer Rückkehrvereinbarung schmackhaft zu machen (Motte 1999). Die Rückkehrförderung wurde allerdings insgesamt nur von einem kleineren Teil der ehemaligen Gastarbeiter in Anspruch genommen. Nach 1990 gerieten viele Arbeitsmigranten - vor allem gering qualifi-

Tabelle 1: Männer des Jahrgangs 1939: Bezieher einer Rente wegen Arbeitslosigkeit (ohne Altersteilzeit)

\begin{tabular}{|c|c|c|c|c|}
\hline & $\begin{array}{l}\text { Deutsche mit } \\
\text { Wohnort in } \\
\text { Westdeutschland }\end{array}$ & $\begin{array}{l}\text { Migranten aus } \\
\text { EU-15 } \\
\text { Anwerbestaaten }\end{array}$ & $\begin{array}{l}\text { Migranten aus } \\
\text { der Türkei und } \\
\text { Ex-Jugoslawien }\end{array}$ & $\begin{array}{l}\text { Deutsche mit } \\
\text { Wohnort in } \\
\text { Ostdeutschland }\end{array}$ \\
\hline $\begin{array}{l}\text { Anteil Rentenbezug wegen } \\
\text { Arbeitslosigkeit an allen } \\
\text { Renten - in \% - }\end{array}$ & 37,2 & 47,1 & 67,3 & 70,1 \\
\hline $\begin{array}{l}\text { Durchschnittliche Rente } \\
\text { bei Rente wegen } \\
\text { Arbeitslosigkeit (Median)* } \\
\text { - in Euro - }\end{array}$ & $1.326,79$ & 910,09 & 761,84 & $1.063,43$ \\
\hline $\begin{array}{l}\text { Anzahl der Bezieher einer } \\
\text { Rente wegen Arbeitslosigkeit }\end{array}$ & 109.233 & 3.728 & 8.303 & 64.208 \\
\hline \multicolumn{5}{|c|}{$\begin{array}{l}\text { Statistik der Deutschen Rentenversicherung - Rentenzugang 1999-2004, nur Jahrgang 1939, eigene Berechnungen. Nur Alters- } \\
\text { renten, nur erstmalige Bewilligung einer Rente, nur Wohnort in Deutschland, keine Aussiedler, keine Nullrenten. } \\
\text { * Rentenzahlbetrag errechnet durch Multiplikation der persönlichen Entgeltpunkte mit dem jeweils gültigen Rentenwert für } \\
\text { Ost- oder Westdeutschland, hochgerechnet auf den 1.7.2007. } \\
\text { Quelle: Berechnungen der Autoren. }\end{array}$} \\
\hline
\end{tabular}

zierte Beschäftigte - durch die stärkere Konkurrenz auf dem westdeutschen Arbeitsmarkt ein weiteres Mal unter Druck, weil Arbeitsuchende aus Ostdeutschland auf den westdeutschen Arbeitsmarkt drängten (Geissler 2004).

\section{Rente wegen Arbeitslosig- keit im Jahrgang 1939}

Die Rente wegen Arbeitslosigkeit wurde und wird von diesem Geburtsjahrgang stark in Anspruch genommen (Wübbeke 2005b, S. 12) (Tabelle 1). An Personen des Jahrgangs 1939 wurde sie dann gezahlt, wenn sie in den letzten 18 Monaten vor dem Alter 60 insgesamt ein Jahr arbeitslos waren. Außerdem mussten in den letzten zehn Jahren vor Rentenbeginn Pflichtbeiträge gezahlt worden sein. Zeiten des Bezugs von Arbeitslosengeld oder Arbeitslosenhilfe wurden berücksichtigt.

Tabelle 1 zeigt, dass bei Männern des Jahrgangs 1939 Arbeitslosigkeit im Alter über 59 Jahren auch unter den im Westen wohnenden Deutschen sehr verbreitet war. In Ostdeutschland und auch bei den beiden untersuchten Migrantengruppen war die Rente wegen Arbeitslosigkeit die häufigste Verrentungsart. Hier spiegelt sich die schlechte Arbeitsmarktlage in Ostdeutschland, aber auch das hohe Arbeitslosigkeitsrisiko von Zuwanderern, vor allem aus der Türkei und aus Ex-Jugoslawien, wider. Zwar entsprach die Frühverrentung unter den ausländischen Arbeitnehmern in den 1990er Jahren in etwa ihrem Anteil an der erwerbstätigen Bevölkerung (Thränhardt 1998, S. 161). Als Alterskohorte waren sie jedoch viel stärker betroffen, weil der Ar- beitsplatzabbau in der Industrie und die Arbeitsplatzrisiken älterer Beschäftigter in dieser Gruppe überdurchschnittlich kumulierten.

Die gleichfalls in Tabelle 1 ausgewiesene durchschnittliche Rente ist ein Spiegel der unterschiedlichen Erwerbskarrieren vor der Arbeitslosigkeit. Die Renten für Westdeutsche sind relativ hoch, was an dem unproblematischen Arbeitseinstieg dieser Alterskohorte und der geringen Arbeitslosigkeit in ihren mittleren Lebensjahren liegt (Kurz 2006, S. 75). Dagegen ist die Rente der Migrantengruppen bereits deutlich niedriger, was vor allem auf ihren späteren Erwerbseintritt und niedrigere Erwerbseinkommen in Deutschland zurückzuführen ist. In dem Geburtsjahrsgang 1939 traten die Migranten aus Italien, Spanien, Portugal und Griechenland im Durchschnitt mit 26 Jahren in den deutschen Arbeitsmarkt ein, die Türken und damaligen Jugoslawen dagegen erst mit 29 Jahren. Im Vergleich zu westdeutschen Männern fehlen ihnen damit durchschnittlich neun Jahre Beitragszahlungen auf ihren Rentenkonten.

Die Analyse der Frühverrentung nach Arbeitslosigkeit für Frauen des Geburtsjahrgangs 1939 (Tabelle 2) ist komplizierter, weil sie sowohl die Rente wegen Arbeitslosigkeit als auch die Altersrente für Frauen in Anspruch nehmen konnten, die beide mit 60 beginnen. Voraussetzung für die Altersrente für Frauen war die Erwerbstätigkeit für mindestens zehn Jahre ab dem 40sten Lebensjahr, also der Wiedereinstieg in den Beruf nach der Familienphase. Weil dies in der DDR die Regel war, erfüllen fast alle Frauen in Ostdeutschland die Voraussetzungen für diese Rente, unabhängig von eventuell auch vorliegender Arbeitslosigkeit.

Anders stellt sich das Bild für Migrantinnen dar. Ihre Renten sind in dieser Ver- 


\begin{tabular}{|c|c|c|c|c|}
\hline & $\begin{array}{l}\text { Deutsche mit } \\
\text { Wohnort in } \\
\text { Westdeutschland }\end{array}$ & $\begin{array}{l}\text { Migranten aus } \\
\text { EU-15 } \\
\text { Anwerbestaaten }\end{array}$ & $\begin{array}{l}\text { Migranten aus } \\
\text { der Türkei und } \\
\text { Ex-Jugoslawien }\end{array}$ & $\begin{array}{l}\text { Deutsche mit } \\
\text { Wohnort in } \\
\text { Ostdeutschland }\end{array}$ \\
\hline $\begin{array}{l}\text { Anteil Rentenbezug in der } \\
\text { Altersrente für Frauen oder } \\
\text { wegen Arbeitslosigkeit im } \\
\text { Vergleich zu allen Altersrenten } \\
\text { - in \% - }\end{array}$ & 51,2 & 68,2 & 70,7 & 94,5 \\
\hline $\begin{array}{l}\text { Durchschnittliche Rente bei } \\
\text { Altersrente für Frauen oder } \\
\text { wegen Arbeitslosigkeit } \\
\text { (Median) }{ }^{*}-\text { in Euro - }\end{array}$ & 727,69 & 582,66 & 530,06 & 778,86 \\
\hline $\begin{array}{l}\text { Anzahl der Bezieherinnen der } \\
\text { Altersrente für Frauen oder } \\
\text { wegen Arbeitslosigkeit }\end{array}$ & 170.936 & 2.476 & 3.379 & 91.919 \\
\hline \multicolumn{5}{|c|}{$\begin{array}{l}\text { Statistik der Deutschen Rentenversicherung-Rentenzugang 1999-2004, nur Jahrgang 1939, eigene Berechnungen. Nur Alters- } \\
\text { renten, nur erstmalige Bewilligung einer Rente, nur Wohnort in Deutschland, keine Nullrenten, keine Aussiedler. } \\
\text { * Rentenzahlbetrag errechnet durch Multiplikation der persönlichen Entgeltpunkte mit dem jeweils gültigen Rentenwert für } \\
\text { Ost- oder Westdeutschland, hochgerechnet auf den 1.7.2007. } \\
\text { Quelle: Berechnungen der Autoren. }\end{array}$} \\
\hline
\end{tabular}

gleichsgruppe niedriger als die der Deutschen in Ost- und Westdeutschland. Ursächlich ist wiederum vor allem der spätere Arbeitsmarkteintritt in Deutschland. Die Frauen aus Italien, Spanien, Portugal und Griechenland zahlten ihren ersten Beitrag zur deutschen Rentenversicherung mit 28 Jahren, die Migrantinnen aus der Türkei und dem damaligen Jugoslawien mit 32 Jahren. Sie waren bei Zuzug nach Deutschland also noch älter als die Männer der gleichen Geburtskohorte.

Bei beiden untersuchten Migrantengruppen erhalten Männer und Frauen gemeinsam gesetzliche Renten, die für einen zwei Personen-Haushalt über Grundsicherungsniveau liegen, und zwar aufgrund der höheren Renten der Männer um etwa $200 €$ bis $300 €$. Die gesetzliche Rente ist für die Haushalte älterer Migranten die wesentliche Einnahmequelle, die nur durch geringe Zusatzeinkommen ergänzt wird (Mika/Tucci 2006b). Dies trifft für ostdeutsche Haushalte ebenfalls zu (Mika/Bieber 2006a), deren Renteneinkommen sich aufgrund ihres früheren Erwerbsbeginns noch günstiger darstellen.

Zusammen mit dem späteren Erwerbseintritt bildet die im Durchschnitt etwa doppelt so hohe Arbeitslosenrate bzw. der

\section{Abb. 1: Überleitung von Arbeitslosigkeit in Altersrente für den Jahrgang 1939}

Lebensverlauf für Geburtsjahrgang 1939 bei Arbeitslosigkeit ab 55 Jahren

Musterfall: geboren am 1.1.1939, 30 Jahre erwerbstätig mit Durchschnittsverdienst, arbeitslos geworden mit 55 Jahren zum 1. Mai 1994. Kein Arbeitsvertrag bis zur Altersrente.

\begin{tabular}{|c|c|}
\hline $\begin{array}{l}\text { Mai 1994-Dezember } 1996 \\
32 \text { Monate Arbeitslosengeld }\end{array}$ & $\begin{array}{l}\text { Januar 1997-Januar } 1998 \\
25 \text { Monate Arbeitslosenhilfe }\end{array}$ \\
\hline $\begin{array}{l}\text { Verlängertes } \\
\text { Arbeitslosengeld für } \\
\text { Personen über } 55 \text { Jahren } \\
\text { - Einkommensersatz in Höhe } \\
\text { von } 60 \% \\
\text { - keine Verpflichtung, deutlich } \\
\text { unter der Qualifikation } \\
\text { Arbeit anzunehmen } \\
\text { - Keine Anrechnung von } \\
\text { Vermögen und Erwerbsein- } \\
\text { kommen im Haushalt (Ver- } \\
\text { sicherungsleistung) }\end{array}$ & $\begin{array}{l}\text { Freistellung vom Arbeits- } \\
\text { markt durch 58er-Regelung } \\
\text { - Einkommensersatz in Höhe } \\
\text { von } 50 \% \text { des letzten Ein- } \\
\text { kommens } \\
\text { - Recht auf } 11 \text { Wochen } \\
\text { Urlaub } \\
\text { - keine Verpflichtung zur } \\
\text { Arbeitssuche } \\
\text { - keine Leistung bei großem } \\
\text { Vermögen und höherem Er- } \\
\text { werbseinkommen des Ehe- } \\
\text { gatten (spezielle bedarfs- } \\
\text { geprüfte Sozialleistung) }\end{array}$ \\
\hline
\end{tabular}

Quelle: Darstellung der Autoren. ab Februar 1999

Altersrente ab 60 Jahren

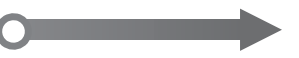

Rente wegen Arbeitslosigkeit oder Rente für Frauen

- Einkommen in Höhe der erreichten Rentenanwartschaften ohne Abschläge (Versicherungsleistung)

WSI MITTELIUNGEN noch häufigere Eintritt in die Altersrente aus Arbeitslosigkeit das Faktorenbündel für die niedrigeren Altersrenten der Arbeitsmigranten.

\section{Abschaffung des Vorruhestands im Kohortenvergleich}

Die Auswirkung der Reformen der Arbeitslosen- und Rentenversicherung soll durch den Vergleich eines identischen Erwerbsund Arbeitslosigkeitsverlaufs in zwei Alterskohorten, 1939 und 1955, verdeutlicht werden. Für die dazwischen liegenden Jahrgänge gelten sowohl im Renten- als auch im Arbeitslosenrecht jeweils Übergangsregelungen, sodass sie schwächer von den Reformen betroffen sind als die 1955 Geborenen. Allerdings bildet die Gruppe der 1955 Geborenen die künftig verschlechterte Einkommenssituation aus der gesetzlichen Rente noch nicht hinreichend ab, da Übergangsfristen der erst 2007 verabschiedeten Erhöhung des Rentenalters auf 67 Jahre mit weiteren Abschlägen von der späteren Rente für Jüngere verbunden sind. Folglich werden spätere Geburtskohorten mit einem noch ungünstigeren Recht konfrontiert sein.

Für unseren Vergleich nehmen wir eine beispielhafte Konstellation:

Der Musterfall, geboren entweder am 1. Januar 1939 oder 1955, wird nach seinem 55. Geburtstag zum 1. Mai arbeitslos und findet bis zum Eintritt in die Rente keine Anstellung mehr. Er hat bis zu diesem Zeitpunkt im Laufe der Erwerbstätigkeit Einkommen in Höhe von dreißig Mal einem Durchschnittsverdienst erzielt und in seiner letzten Beschäftigung durchschnittlich verdient. Die Musterperson hat Anspruch auf Leistungen der Bundesagentur für Arbeit nach dem jeweils geltenden Recht.

Für den Jahrgang 1939 sah die Überleitung für den oben genannten Musterfall folgendermaßen aus (Abbildung 1):

An den verlängerten Anspruch auf Arbeitslosengeld von 32 Monaten schloss sich der Bezug von Arbeitslosenhilfe an, der zwar einer Bedarfsprüfung unterlag, die jedoch gerade für Ältere deutlich großzügiger gestaltet war als die der Sozialhilfe. Nicht nur lagen die Vermögensfreigrenzen 
erheblich höher, sodass die im Lauf des Erwerbslebens angesparten Vermögen in der Regel nicht angetastet wurden (Mika 1999, S. 436), sondern auch das Erwerbseinkommen des Ehepartners wurde nur zum Teil in die Berechnung der Bedürftigkeit einbezogen. Die großzügige sozialstaatliche Absicherung für ältere Arbeitslose erscheint als sozialpolitische Kehrseite der geringen Fähigkeit des deutschen Beschäftigungssystems, ältere Beschäftigte in einer sich verändernden Arbeitswelt weiterzubilden (Buchholz 2006, S. 55).

Der biografische Ablauf ändert sich für Musterbiografien des Jahrgangs 1955 tief greifend, zunächst durch die Reform der Arbeitslosenversicherung. Das Arbeitslosengeld wird kürzer, nur noch für $18 \mathrm{Mo-}$ nate gezahlt. Die Arbeitslosenhilfe wurde 2005 abgeschafft und durch die strenger geprüfte und in der Höhe meist geringere Zahlung von ALG II ersetzt, das keine Lohnersatzleistung mehr darstellt. Der Ablauf stellt sich nun wie in Abbildung 2 gezeigt dar.

Der größte Einschnitt für ältere Arbeitslose ist der Wegfall der sogenannten 58er Regelung, die eine Freistellung von der Verpflichtung zur Arbeitssuche bedeutete. Die Phase der Arbeitslosigkeit bei Bezug bedürfnisgeprüfter Arbeitslosensicherung dauert im Jahrgang 1955 länger, weil sie zum einen früher beginnt und weil zum anderen das frühestmögliche Verrentungsalter von 60 Jahren auf 63 Jahre erhöht wurde.

\section{Abgesenkte Rente wegen Abschaffung des Vorruhestands}

Die Reform der Arbeitslosen- und Rentenversicherung hat auch Folgen für die Höhe der späteren Rente von Langzeitarbeitslosen. Einerseits werden seit 2000 sukzessive immer weniger Beiträge für Langzeitarbeitslose an die Rentenversicherung abgeführt, sodass in den persönlichen Rentenkonten geringere Anwartschaften aufgebaut werden. ${ }^{1}$ Andererseits werden nun Abzüge für die Inanspruchnahme der Rente vor dem Erreichen der Regelaltersrente vorgenommen, die der Musterfall der Alterskohorte 1939 nicht hinnehmen musste. ${ }^{2}$ Tabelle 3 zeigt, wie sich eine Altersrente für den Musterfall 1939 berechnet, zum Vergleich hochgerechnet auf das Jahr 2007.

Tabelle 3: Rentenberechnung für den Musterfall Geburtsjahrgang 1939

\begin{tabular}{|lccl|}
\hline & Entgeltpunkte & $\begin{array}{c}\text { Rentenbetrag } \\
- \text { in Euro - }\end{array}$ & Anmerkungen \\
\hline $\begin{array}{l}\text { Rentenbeiträge aus } \\
\text { Erwerbstätigkeit }\end{array}$ & 30,0 EGPT & 788,10 & $\begin{array}{l}\text { Beiträge aus Durchschnittsverdienst } \\
\text { bringen pro Jahr einen Entgeltpunkt (EGPT) } \\
\text { in der Rentenversicherung }\end{array}$ \\
\hline Beiträge aus ALG I & 2,2 EGPT & 57,79 & $\begin{array}{l}\text { Während der 32 Monate im Bezug von } \\
\text { Arbeitslosengeld wurden Beiträge in Höhe } \\
\text { von 80 \% des letzten Jahresverdienstes an } \\
\text { die Rentenversicherung gezahlt }\end{array}$ \\
\hline Beiträge aus AlHi & 1,6 EGPT & 42,04 & $\begin{array}{l}\text { Auch in den 24 Monaten Arbeitslosenhilfe } \\
\text { wurden Beiträge wie beim ALG entrichtet }\end{array}$ \\
\hline Summe & 33,8 EGPT & 887,93 & \\
\hline $\begin{array}{l}\text { Abschläge wegen } \\
\text { Renten ab 60 Jahren } \\
- \text { in \% - }\end{array}$ & 0 & $\begin{array}{l}\text { Es wurden keine Abschläge für } \\
\text { frühere Inanspruchnahme erhoben }\end{array}$ \\
\hline Quelle: Berechnung der Autoren mit dem aktuellen Rentenwert West ab 1.7.2007 (26,27 €). WSI MITTEILUNGEN
\end{tabular}

Abb. 2: Lebensphase zwischen Arbeitslosigkeit und Altersrente für den Jahrgang 1955

Lebensverlauf für Geburtsjahrgang 1955 bei Arbeitslosigkeit ab 55 Jahren

Musterfall: geboren am 1.1.1955, 30 Jahre erwerbstätig mit Durchschnittsverdienst, arbeitslos geworden mit 55 Jahren zum 1. Mai 2010. Kein Arbeitsvertrag bis zur Altersrente.

\begin{tabular}{|c|c|c|}
\hline $\begin{array}{l}\text { Mai 2010-Oktober } 2011 \\
18 \text { Monate ALG I }\end{array}$ & $\begin{array}{l}\text { November 2011-Januar } 2018 \\
6 \text { Jahre } 3 \text { Monate ALG II }\end{array}$ & $\begin{array}{l}\text { ab Februar } 2018 \\
\text { Altersrente ab } 63 \text { Jahren }\end{array}$ \\
\hline $\begin{array}{l}\text { Verlängertes Arbeits- } \\
\text { losengeld für Personen über } \\
55 \text { Jahren } \\
\text { - Einkommensersatz in Höhe } \\
\text { von } 60 \% \text { des vorherigen } \\
\text { Einkommens } \\
\text { - keine Verpflichtung, deutlich } \\
\text { unter der Qualifikation } \\
\text { Arbeit anzunehmen } \\
\text { - Keine Anrechnung von } \\
\text { Vermögen und Erwerbsein- } \\
\text { kommen im Haushalt }\end{array}$ & $\begin{array}{l}\text { - Bedarfsgeprüftes Mindest- } \\
\text { einkommen } \\
\text { - Verpflichtung, dem Arbeits- } \\
\text { markt zur Verfügung zu } \\
\text { stehen } \\
\text { - keine Leistung bei geringem } \\
\text { Vermögen oder ausrei- } \\
\text { chendem Erwerbseinkom- } \\
\text { men des Ehegatten für } \\
\text { Lebensstandard auf Grund- } \\
\text { sicherungsniveau (bedarfs- } \\
\text { geprüfte Leistung) }\end{array}$ & $\begin{array}{l}\text { Rente für langjährig } \\
\text { Versicherte } \\
\text { - Rente in Höhe der erreich- } \\
\text { ten Rentenantwartschaften } \\
\text { abzüglich 9,6\% Abschläge } \\
\text { (Versicherungsleistung) }\end{array}$ \\
\hline Ruelle: Darstellung der Autoren. & & WSI mitteILUNGEN \\
\hline
\end{tabular}

Für die Personen des Jahrgangs 1955 mit einer identischen Musterbiografie werden nun weitaus geringere Beiträge an die Rentenversicherung gezahlt. Außerdem werden Abschläge für die Inanspruchnahme einer Rente vor dem Erreichen der Regelaltersrente erhoben (Nakielski/Kerschbaumer 2007, S. 86). Damit erreichen die Personen mit der Musterbiografie des Jahrgangs 1955 deutlich geringere Rentenzahlungen, trotz des späteren Rentenbeginns (Tabelle 4). ${ }^{3}$

Insgesamt addieren sich die drei Faktoren, die zu einer niedrigeren Rente führen, zu einer um rund ein Drittel $(32,1 \%)$ niedrigeren Rente im Kohortenvergleich. Dies ist das Ergebnis der angesprochenen Regelungen, d.h. dass hier nicht alle Kürzungsfaktoren der Rentenreformen berücksich- tigt sind. Die niedrigeren Beiträge aus der Zeit der Arbeitslosigkeit, die Abschläge bei vorzeitiger Rente und der Riester- und Nachhaltigkeitsfaktor tragen in nahezu gleichen Teilen zu diesem Ergebnis bei.

\footnotetext{
1 Die Beträge wurden in mehreren Schritten gesenkt. Die Bezugsgröße für die Rentenbeiträge wurde 2000 auf die Höhe der gezahlten Arbeitslosenhilfe, also um $30 \%$, herabgesetzt. Ab 2005 wurden noch $78 €$ monatlich, ab 2007 nur noch $40 €$ monatlicher Beitrag abgeführt.

2 Zwar waren auch für den Jahrgang 1939 Abzüge bei der Rente wegen Arbeitslosigkeit vorgesehen, gem. § 237 Abs. 4 S. 1 SGB VI hätte unser Musterfall aber Vertrauensschutz genossen, weil er vor dem 14.2.1996 (Stichtag) arbeitslos wurde. Dies galt für alle bis zum 14.2.1941 Geborenen, die am Stichtag arbeitslos waren.

3 Um den Vergleich zu ermöglichen, wird ebenfalls mit den Werten von 2007 gerechnet.
} 
Ausmaß arbeitslos waren und sind. In den neuen Bundesländern gibt es den langen, prekären Altersübergang aus mehrjähriger Arbeitslosigkeit oder sozial vergleichbarer Lage - wie Nichterwerbstätigkeit oder Krankheit - in den letzten Rentenzugangsjahren ebenso häufig wie den bruchlosen Rentenzugang aus Erwerbstätigkeit (Brussig/Wojtkowski 2007, S. 95; Brussig et al. in diesem Heft).

Der Ausbau der Rentenversicherung für Arbeiter und Angestellte und die Verlängerung der Lebensphase im Ruhestand für alle sozialen Schichten waren einst zentrale sozialstaatliche Maßnahmen zum Abbau sozialer Ungleichheit in der deutschen Gesellschaft. Der Vorruhestand, einschließ- lich der großzügigen Regelungen der Arbeitslosensicherung, war ein Bestandteil dieses Institutionengefüges, der besonders für viele Ostdeutsche nach der Wiedervereinigung und für Industriearbeiter in Westdeutschland über viele Jahre hinweg einen sicheren Einkommensersatz bot. Das milderte insbesondere die Einkommensrisiken Un- und Angelernter, die ab dem 55. Lebensjahr ungleich häufiger von Arbeitslosigkeit betroffen sind. Sie konnten den Übergang in den Ruhestand vorziehen und die Rente durch weitere Beiträge in Phasen von Arbeitslosigkeit und Krankheit so erhöhen, dass sich Langzeitarbeitslosigkeit nicht durchschlagend auf den Lebensstandard im Alter auswirkte. Durch die struk- turellen Veränderungen der Arbeitslosenversicherung müssen die älteren Arbeitslosen nun durch eine biografisch lange, sozial unsichere Zwischenperiode gehen, in der sie nur bei insgesamt sehr ungünstiger ökonomischer Lage im Haushalt Unterstützung durch ALG II erhalten. Deshalb nehmen bisher die meisten Betroffenen lieber Abzüge für eine möglichst frühe Inanspruchnahme der Rente in Kauf (Brussig/ Wojtkowsi 2007; neueste Auswertungen vgl. Brussig et al. in diesem Heft), als auf eine ungekürzte Altersrente mit 65 Jahren zu warten. Diese Option bietet sich den später Geborenen nicht mehr.

\section{LITERATUR}

Albrecht, G./Müller, H. W. (1996): Die neue Altersrente wegen Arbeitslosigkeit oder nach Altersteilzeit, in: Deutsche Rentenversicherung 2, S. 121-144

Brall, N./Fasshauer, S./Lübke, E./Stahl, H. (2004): Neuregelungen im Bereich der Altersgrenzen, DRV-Schriften 350

Brenke, K. (2007): Die Bedeutung der Älteren auf dem Arbeitsmarkt nimmt deutlich zu, DIW-Wochenbericht 74, S. 337-345

Brussig, M./Wojtkowski, S. (2007): Immer mehr Neurentner müssen Abschläge hinnehmen, in: Soziale Sicherheit 8, 2007, S. 90-96

Buchholz, S. (2006): Men's late careers and career exits in West Germany, in: Blossfeld, H.P/Buchholz, S./Hofäcker, D. (Hrsg.): Globalisation, Uncertainty and late careers in Society, London, S. 55-78

Buchholz, S./Hofäcker, D./Blossfeld, H.-P. (2006): Globalization, accelerating economic change and late careers, in: Blossfeld, H.P./Buchholz, S./ Hofäcker, D. (Hrsg.): Globalisation, Uncertainty and late careers in Society, S. 1-23

Fasshauer, S. (2005): Die Folgen des demographischen Wandels für die gesetzliche Rentenversicherung, in: Kerschbaumer, J./Schroeder, W. (Hrsg.): Sozialstaat und demographischer Wandel. Herausforderungen für Arbeitsmarkt und Sozialversicherung, Wiesbaden, S. 67-95

Gasche, M. (2007): Intergenerative Verteilungseffekte der (modifizierten) Schutzklausel und der Anhebung des gesetzlichen Renteneintrittsalters, in: Deutsche Rentenversicherung 2/3, S. 158-169

Geissler, R. (2004): Die Sozialstruktur Deutschlands. Zur gesellschaftlichen Entwicklung mit einer Bilanz zur Vereinigung, Wiesbaden Kurz, K./Hillmert, S./Grunow, D. (2006): Increasing instability in employment careers of West German men? A comparison of the birth cohorts 1940, 1955 and 1964, in: Blossfeld, H. P./Mills, M./Bernardi, F.: Globalisation, Uncertainty, and Men's Career. An international comparison, Cheltenham, S. 75-113
Maier, H./Scholz, R. (2004): Wiedervereinigung zeigt: Für ein langes Leben ist es nie zu spät. Ostdeutsche holen in der Lebenserwartung auf. Demografische Forschung aus erster Hand 1, S. 1-2

Mika, T. (1999): Siebter Senat des Bundessozialgerichts nimmt zur Lebensstandardsicherung Stellung, in: Deutsche Angestellten Versicherung 8, S. 434-436

Mika, T./Bieber, U. (2006a): Verdeckte Armut der älteren Bevölkerung. Ausmaß von Niedrigeinkommen und Gründe der Nichtinanspruchnahme von Sozialhilfe unter Senioren, in: Deutsche Rentenversicherung 4-5, S. $248-278$

Mika, T./Tucci I. (2006b): Alterseinkommen bei Zuwanderern. Gesetzliche Rente und Haushaltseinkommen bei Aussiedlern und Zuwanderern aus der Türkei und dem ehemaligen Jugoslawien im Vergleich zur deutschen Bevölkerung, in: Deutsche Rentenversicherung 7-8, S. 456-483 Moen, Ph. (2004): Midcourse. Navigating retirement and a New Life Stage, in: Jeylan T. M./Shanahan, M. J./Mortimer. J. (Hrsg.): Handbook of the life course, New York, S. 269-291

Motte, J. (1999): Gedrängte Freiwilligkeit. Arbeitsmigration, Betriebspolitik und Rückkehrförderung 1983/84, in: Motte, J./Ohliger, R./Oswald, A. von (Hrsg.): 50 Jahre Bundesrepublik - 50 Jahre Einwanderung. Nachkriegsgeschichte als Migrationsgeschichte, Frankfurt/M./New York, S. 165-183 Nakielski, H./Kerschbaumer, J. (2007): Bundestag verabschiedet "Rente mit $67^{\prime \prime}$. Die neuen Altersgrenzen bei den Rentenarten, in: Soziale Sicherheit 3, S. 85-89 OECD (2007): Pensions at a glance, Paris

Promberger, M./Wübbeke, C. (2006): Anhebung der Regelaltersgrenze. Pro und Contra Rente mit 67, IAB Kurzbericht 8, S. 1-5 
Ruland, F., (2007): Die gesetzliche Rentenversicherung im Wandel der Herausforderungen - Zentrale Reformen nach 1957 bis 2007, in: Deutsche Rentenversicherung Bund: Die gesetzliche Rente in Deutschland 50 Jahre Sicherheit durch Anpassungen, Bad Homburg, S. 29-46

Schmähl, W. (2007): Die Einführung der Dynamischen Rente im Jahr 1957: Gründe, Ziele und Maßnahmen - zugleich Versuch einer Bilanz nach 50 Jahren, in: Deutsche Rentenversicherung Bund: Die gesetzliche Rente in Deutschland - 50 Jahre Sicherheit durch Anpassungen, Bad Homburg, S. 9-28

Seifert, W. (2000): Geschlossene Gesellschaften - offene Grenzen? Migrations- und Integrationsprozesse in westlichen Industrienationen, Frankfurt/New York

Statistisches Bundesamt (Hrsg.) (2006): Datenreport 2006. Zahlen und Fakten über die Bundesrepublik Deutschland, Bundeszentrale für politische Bildung

Stegmann, M. (2008): Typisierung von Versicherungsverläufen - Eine explorative Analyse auf der Basis der Versicherungskontenstichprobe 2005, in: Deutsche Rentenversicherung 2, S. 221
Tesch-Römer, C./Wurm, S. (2006): Veränderung des subjektiven Wohlbefindens in der zweiten Lebenshälfte, in: Tesch-Römer, C./Engstler, H./ Wurm, S. (Hrsg.): Älterwerden in Deutschland. Sozialer Wandel und individuelle Entwicklungsverläufe, S. 385-446

Thränhardt, D./Schäfer, M. (1998): Inklusion und Exklusion: Die Italiener in Deutschland, in: Thränhardt, D. (Hrsg.): Jahrbuch Migration 1997/1998, Osnabrück, S. 149-179

Trappe, H. (2006): Lost in Transformation? Disparities of Gender and Age, in: Diewald, M./Goedicke, A./Mayer, K. U.: After the fall of the Wall, Stanford, S. 116-139

Whitehouse, E. (2006): New Indicators of 30 OECD countries' pension systems, in: Journal of pension economics and finance 3, S. 275-298 Wübbeke, C. (2005a): Der Übergang in den Rentenbezug im Spannungsfeld betrieblicher Personal- und staatlicher Sozialpolitik. Beiträge zur Arbeitsmarkt- und Berufsforschung 290.1, Nürnberg IAB

Wübbeke, C. (2005b): Der Übergang in den Rentenbezug im Spannungsfeld betrieblicher Personal- und staatlicher Sozialpolitik. Anhangband, Beiträge zur Arbeitsmarkt- und Berufsforschung, 290.2, Nürnberg IAB 